\title{
A Multifrequency Radio Spectral Study of SNR HB21
}

\author{
X.Z. Zhang ${ }^{1,2}$, L.A. Higgs ${ }^{3}$, T.L. Landecker ${ }^{3}$,S.J. Qian ${ }^{1,2}$ and X.J $\mathrm{Wu}^{4}$ \\ 1 Beijing Astronomical Observatory, CAS, Beijing 100012, China \\ 2 National Astronomical Observatories, CAS, Beijing 100012, China \\ ${ }^{3}$ Dominion Radio Astrophysical Observatory, P.O.Box 248, Penticton, \\ B.C. V2A 6K3, Canada \\ ${ }^{4}$ Department of Geophysics, Peking University, 100871, China
}

\begin{abstract}
Radio observational results at $232 \mathrm{MHz}$ and multifrequency studies of supernova remnant(SNR) HB21 are presented in this paper. Both the integrated spectral index and the spatial variations of spectral index of the remnant were calculated by combining the new map at 232 $\mathrm{MHz}$ with previously published maps made at 408, 1420, 2695, and 4750 $\mathrm{MHz}$.
\end{abstract}

\section{Observation}

HB21(G89.0+4.7) is an evolved supernova remnant having an angular size of about $1.5^{\circ}(N S) \times 2.0^{\circ}(E W)$. New observations with high resolution at 232 $\mathrm{MHz}$ were carried out in June, 1992 with the Miyun Synthesis Radio Telescope(MSRT), Beijing Astronomical Observatory which has 192 baselines from $18 \mathrm{~m}$ to $1164 \mathrm{~m}$ with the interval of $6 \mathrm{~m}$. The missing baselines of $0,6,12$ $m$ cause a weak large negative background which affects the measurement of total intensity and large scale structure of HB21. A method has been developed (Zhang 1995) to reduce the effects of missing short spacings by using interferometer data only, following Braun and Walterbos (1985). The data were calibrated using Cygnus A and 3C418 and 3C418 was estimated to hace a flux density of $13.2 \pm 0.6 \mathrm{Jy}$ in the BGPW scale.

\section{Results and Discussions}

The total intensity map of HB21 at $232 \mathrm{MHz}$ shown in Fig. 1(a) has an integrated flux density of $450 \mathrm{Jy}$ at $232 \mathrm{MHz}$. The structure agrees very well with that obtained from other frequencies. By combining our integrated flux with that at $4750 \mathrm{MHz}(110 \pm 5 \mathrm{Jy})$ from the maps of Reich(1983), the integrated spectral index is -0.47 between $232-4750 \mathrm{MHz}$.

Spectral spatial variations of HB21 between $232,408,2695$ and $4750 \mathrm{MHz}$ have been calculated using $\mathrm{T}-\mathrm{T}$ plots with all maps convolved to a resolution

\footnotetext{
${ }^{1}$ The project is supported by the National Natural Science Foundation of China and the National Climbing programme of China.
} 

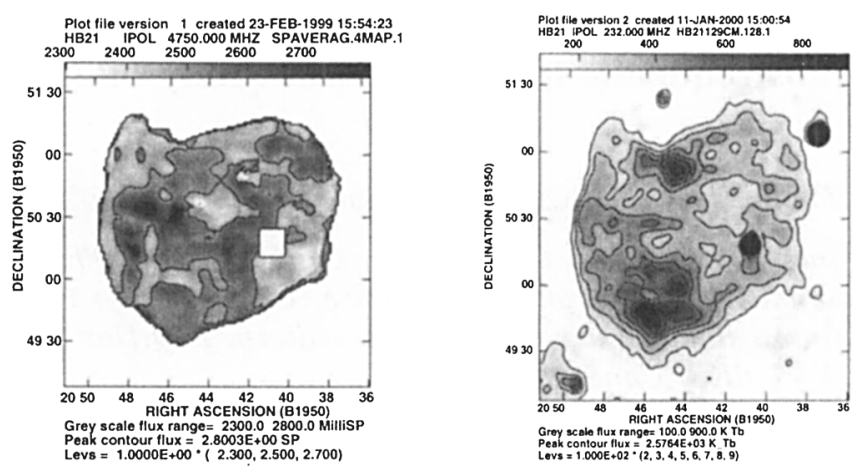

Figure 1. The left (a) is the temperature map of HB21 at $232 \mathrm{MHz}$ and the right (b) is the frequency-average spectral index map of HB21.

of $5.2^{\prime} \times 4.7^{\prime}$ and the average spectral index map is shown in Fig. 1(b). The spectral map shows that : (1) the spectral index on scales larger than $10^{\prime}$ is 0.55 at southeast, falling to 0.35 at northwest; (2)in the central area of the remnant, the median of spectral index is about 0.3 , whereas the average spectral index is about 0.47 .

Tatematsu et al. (1990) have detected HI and CO along the east and south boundary and HB21 is interacting with this gas. The structure of the central flat spectrum region agrees well with the structure of X-ray seen by ROSAT but not with the optical filaments, which could be caused by post-shock reheating of the interstellar medium.

The Galactic background emission is probably another reason for the large scale spectral variations from the southeast to the northwest of HB21, as the direction along which the spectral index decreases, is nearly in the direction of the increasing Galactic latitude. Milne (1987) showed that HB21 has a magnetic field sweeping across the remnant from north-east to south-west.

The phenomenon of spectra flattening near the edges obtained from our new result is similar to that from SNR HB9 (Leahy, et al. 1999). Both HB9 and HB21 are old and evolved supernova remnants. The electron density integrated along the line-of-sight is highest inside the rim. So absorption by the thermal electrons will be strong there. This causes spectral flattening at low frequency.

\section{References}

Braun, R., Walterbos, R.A.M. 1985,A\&A, 143, 307

Leahy, D.A., Zhang X.Z., Wu X.J., \& Lin J.L. 1998,A\&A,339,601

Milne, D.K. 1987, Aust. J. Phys., 40, 771

Tatematsu, K., Fukui, Y., Landecker, T.L., \& Roger, R.S. 1990, A\&A,1990, 237, 189

Reich, W., Furst, E. \& Sieber 1983, in proceedings of IAU Symp. 101, 377

Zhang, X.Z. 1995, Annals of Shanghai Observatory, p291 